\title{
Efficacy and Safety of Tadalafil Once-a-Day versus Tadalafil On-Demand in Patients with Erectile Dysfunction: A Systematic Review and Meta-Analyses
}

\author{
Zhufeng Peng Lu Yang Qiang Dong Qiang Wei Liangren Liu Bo Yang \\ Department of Urology, Institute of Urology, West China Hospital, Sichuan University, Chengdu, China
}

\section{Keywords}

Dosing regimen · Erectile dysfunction · Systematic review · Tadalafil

\begin{abstract}
Introduction: To compare the efficacy and safety between tadalafil once-a-day and tadalafil on-demand dosing regimen in patients with ED. Materials and Methods: A systematic search of Medline, Embase, and Cochrane Library was performed to identify all randomized controlled trials (RCTs) that compared tadalafil used a once-a-day with an on-demand dosing regimen for erectile dysfunction. A secondary hand-search was performed in relevant journals, references, and the grey literature. Meta-analyses were performed using Review Manager version 5.3.0. Results: Six RCTs involving a total of 1,534 patients were included in this review. All studies reported the International Index of Erectile FunctionErectile Function domain score and the results of the metaanalysis showed no difference between the groups. The overall pooled estimated weighted mean differences (WMD) was 0.97 (95\% Cl -0.37 to $2.32 ; p=0.16)$. Meta-analyses of Sexual Encounter Profile questions 2 and 3 (SEP-2 and SEP-3) showed that the once-a-day dosing regimen was superior to the on-demand regimen with statistical significance. The WMD of SEP-2 and SEP-3 were 10.32 (95\% Cl 3.16-17.48; $p=$ $0.005)$ and 11.07 (95\% Cl 2.57-19.56; $p=0.01)$, respectively. Both dosing regimens of tadalafil showed similar complication rates. The meta-analyses of adverse events showed no significant differences. Conclusions: The efficacy rates of
\end{abstract}

\section{KARGER}

(C) 2017 S. Karger AG, Basel

E-Mail karger@karger.com

www.karger.com/uin tadalafil once-a-day and on-demand were similar. No significant difference in safety was found between the 2 dose regimens of tadalafil.

(c) 2017 S. Karger AG, Basel

\section{Introduction}

Erectile dysfunction (ED) is defined as the persistent inability to attain or maintain an erection sufficient for satisfactory sexual performance [1]. ED is a common problem among men worldwide. Several studies have demonstrated that the prevalence of ED varies from 37.5 to $52 \%$ in men between 40 and 70 years of age, with an incidence rate of new cases between 1.9 and $2.6 \%$ annually [2].

ED is a complex condition that may be associated with many factors, including endocrine disorders, metabolic disorders, cardiovascular disease, psychogenic disorders, or major surgery of the pelvis [3]. Apart from treating the diseases that cause ED, the treatment of ED also includes oral pharmacotherapy, intracavernous injections, intraurethral and topical pharmacotherapy, vacuum erection devices, and penile prosthesis implantation [4]. Among these, phosphodiesterase 5 (PDE5) inhibitors have been widely used as first-line treatments for ED because of their well-established efficiency, safety, and convenience parameters. PDE5 inhibitors can decrease the degradation of cyclic guanosine monophosphate leading to smooth muscle relaxation and increased blood flow,

Qiang Wei and Qiang Dong

Department of Urology

West China Hospital of Sichuan University

No. 37 Guoxue Xiang, Chengdu 610041, Sichuan (China)

E-Mail weiqiang933@126.com; dong_qiang@ mcwcums.com 
which result in penile erection [5]. Currently, 4 selective PDE5 inhibitors have been approved by the European Medicines Agency for the treatment of ED, including sildenafil, tadalafil, vardenafil, and avanafil [6].

PDE5 inhibitors are commonly used as an on-demand treatment regimen because of their short duration of efficacy. However, tadalafil, unique among other PDE5 inhibitors, has demonstrated efficacy up to $36 \mathrm{~h}$ and is not affected by the intake of food [7]. Thus, tadalafil could be taken regularly, such as once a day, to provide continuous efficacy, which would enable patients to engage in sexual intercourse at any time, eliminating the need to take a dose before intercourse [8].

Different dosing regimens of tadalafil have demonstrated significant efficacy and have been well-tolerated in randomized placebo-controlled trials and in integrated analysis studies of ED patients. However, there is controversy concerning which dosing regimen of tadalafil is optimal - on-demand or once-a-day. We undertook this systematic review to compare the efficacy and safety of different dosing regimens of tadalafil in patients with ED.

\section{Materials and Methods}

\section{Search Strategy}

Literature searches of electronic databases were performed in April 2017. We searched the databases of Medline, Embase, and Cochrane Central Register of Controlled Trials (CENTRAL) to identify a randomized controlled trial (RCT) that referred to the impact of different dosing regimens of tadalafil in patients with ED. The following key terms were used as MeSH terms and free text: "tadalafil," "erectile dysfunction," "randomized," "on demand," "as needed," "once a day" and "daily." Multiple synonyms of each term were also searched. The Medline search strategy was presented in Appendix 1. The same search strategies were used for Embase and CENTRAL. There was no language restriction. All titles and abstracts retrieved by electronic searching were screened by 2 reviewers (Z.P. and L.Y.) in duplicate. Full-text publications were reviewed for identifying all potentially eligible studies. Relevant journals, references, and greyliterature papers were hand-searched.

\section{Selection Criteria}

All studies were screened on the basis of the following criteria: the study patients were at least 18 years of age and had received a diagnosis of ED; the study evaluated the efficacy or safety of tadalafil once-a-day versus on-demand for ED and was conducted using an RCT design; and full texts of English articles were available. Studies that met all criteria were included. Multiple articles from identical trials were regarded as one study. Only the most detailed article was included.

\section{Data Extraction}

Data extraction was performed by 2 authors (Z.P. and L.L.) in duplicate. The following information was collected, including study characteristics, study designs, participant characteristics, in- terventions, follow-up, outcomes, and limitations of the study. Our primary outcomes included the International Index of Erectile Function-Erectile Function (IIEF-EF) domain score. Secondary outcomes included Sexual Encounter Profile question 2 (SEP-2): "Were you able to insert your penis into your partner's vagina?," Sexual Encounter Profile question 3 (SEP-3): "Did your erection last long enough for you to have successful intercourse?," Treatment Preference Question: "Which treatment do you prefer?" and adverse events. When the studies information was incomplete, we contacted the authors for missing data.

\section{Quality Assessment}

The quality of included studies was assessed by 2 authors (Q.D. and B.Y.) independently. Discrepancies were resolved in consultation with the third reviewer (Q.W.). The quality of RCTs was assessed by the Cochrane Risk of Bias Tool, which included 7 specific domains: random sequence generation, allocation concealment, blinding of participants and personnel, blinding of outcome assessment, incomplete outcome data, selective reporting, and other sources of bias [9]. Judgments were assigned using one of the 3 categories: "low risk" of bias, "high risk" of bias, or "unclear risk" of bias.

\section{Statistical Analyses}

All meta-analyses were performed using Review Manager version 5.3.0 (The Cochrane Collaboration, Oxford, UK). Continuous data were assessed by weighted mean differences (WMD), and dichotomous data were assessed by relative risk with $95 \%$ CIs. Heterogeneity among studies was assessed with the chi-squared test and the I 2 index statistic. $p$ values $<0.1$ or $I^{2}>40 \%$ were considered, heterogeneity existed, and random-effects models were then used for meta-analysis. Otherwise, if there were no important heterogeneity among the studies, fixed-effects models were used. When heterogeneity was present, subgroup analyses were performed. Sensitivity analysis was used to verify the reliability of the results.

\section{Results}

\section{Results of the Systematic Literature Review and Study Characteristics}

A systematic search of databases was performed in April 2017. We initially identified 554 articles. After screening titles and abstracts, 543 duplicates and irrelevant articles were ruled out. After full-text assessment of 11 articles, 6 RCTs involving a total of 1,534 patients were included in this review (Fig. 1). Among these, 4 RCTs [10-13] included populations of broad-spectrum ED patients. Two RCTs $[14,15]$ investigated prostate cancer patients with ED. One RCT analyzed prostate cancer patients with post-radiotherapy ED. One RCT studied prostate cancer patients with post-NSRP (nerve-sparing radical prostatectomy) ED. All RCTs compared tadalafil using a once-a-day with an on-demand dosing regimen for ED. Characteristics of the included studies are shown in Table 1. Risk-of-bias assessments of the included studies are presented in Figure 2. 


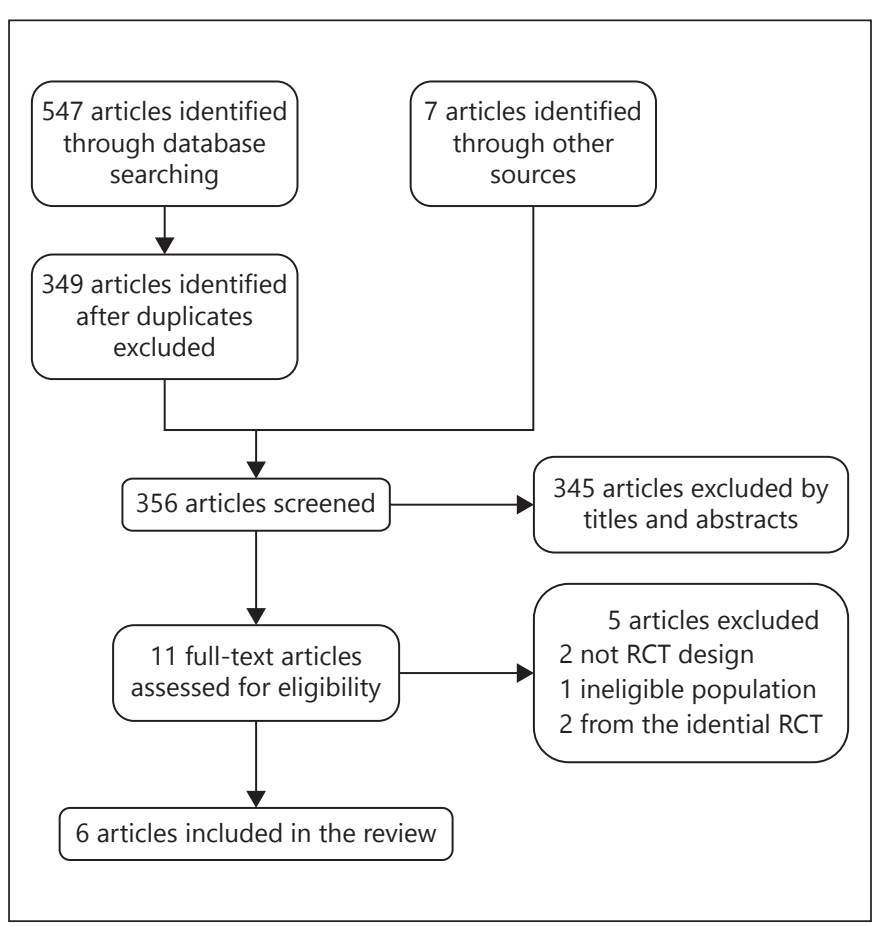

Fig. 1. Flowchart of inclusion of studies.

\section{IIEF-EF Domain Score}

All RCTs reported IIEF-EF domain score changes from baseline and showed that treatment with tadalafil on-demand or once-a-day significantly improved erectile function from baseline. All studies were included in the meta-analysis. Both clinical and statistical heterogeneity were presumed to exist among the trials. Therefore, subgroup analysis was conducted according to different patient population. The overall pooled estimated WMD was 0.97 (95\% CI -0.37 to $2.32 ; p=0.16$ ). The pooled WMD of broad-spectrum ED patients and prostate cancer ED patients showed similar results. The WMD was 0.84 (95\% CI -0.81 to $2.50 ; p=0.32$ ) for broad-spectrum ED patients and $1.50(95 \% \mathrm{CI}-0.56$ to $3.55 ; p=0.15)$ for prostate cancer ED patients. The overall and subgroup analysis showed no significant difference between oncea-day and on-demand dosing regimen in the IIEF-EF score. The meta-analysis of IIEF-EF scores is presented in Figure 3.

\section{SEP-2 and SEP-3}

Three studies $[12,14,15]$ that assessed the mean perpatient percentage of "yes" responses to SEP-2 with useable data were included in the meta-analysis. Heterogeneity was found among the studies. Subgroup analysis was also conducted according to different patient

Tadalafil Once-a-Day vs. Tadalafil

On-Demand for ED population. The overall pooled estimated WMD was 10.32 (95\% CI 3.16-17.48; $p=0.005$ ). The WMD of broad-spectrum ED patients was 12.00 (95\% CI 0.05$23.95 ; p=0.05)$. The pooled WMD of prostate cancer ED patients was 9.38 (95\% CI 0.44-18.32; $p=0.04)$. Two studies $[12,14]$ that assessed the mean per-patient percentage of "yes" responses to SEP-3 were included in the meta-analysis. The overall pooled WMD was 11.07 (95\% CI 2.57-19.56; $p=0.01$ ). The meta-analyses of SEP-2 and SEP-3 are presented in Figure 4. Among these studies that reported SEP-2 or SEP-3, the results were similar to the respective outcome of the IIEF-EF domain score.

\section{Treatment Preference Question}

One of the studies reported Treatment Preference Question of different dosing regimens of tadalafil in the treatment of ED. In a study conducted by McMahon [12], 105 patients (72\%) preferred tadalafil once-a-day and 40 patients (28\%) preferred tadalafil on-demand. The reasons for preference for once-a-day regimen were superior sexual spontaneity (55.2\%), superior efficacy (30.5\%), and fewer adverse events (11.4\%). The reasons for preference for on-demand regimen were no perceived advantage of once-a-day tadalafil (32.5\%), concerns of risks of long-term use of tadalafil once a day (25\%), superior efficacy (12.5\%), and fewer adverse events $(7.5 \%)$.

\section{Adverse Events}

Adverse events of tadalafil were relatively rare. Most adverse events were reported as mild and well tolerated. Only $2.3 \%$ patients $(48 / 2,022)$ discontinued the studies due to intolerable adverse events. The percentages of patients who discontinued due to adverse events were similar among the tadalafil once-a-day group $(2.7 \%, 27 / 1,010)$ and the on-demand group $(2.1 \%, 21 / 1,012)$. The overall common adverse events reported included the following: headache, dyspepsia, flushing, backache, and myalgia. These 5 adverse events were analyzed by meta-analysis. No heterogeneity was observed in all adverse event analyses. The use of tadalafil once-a-day and on-demand showed similar complication rates. The pooled relative risks of all adverse events showed no significant differences. The meta-analyses of adverse events are presented in Figure 5.

McMahon [12] reported that 4 patients with significant multiple vascular risk factors had acute myocardial infarctions, 2 patients were in the on-demand group and 2 were in the once-a-day group. Montorsi et al. [14] re-

Urol Int 2017;99:343-352 345 


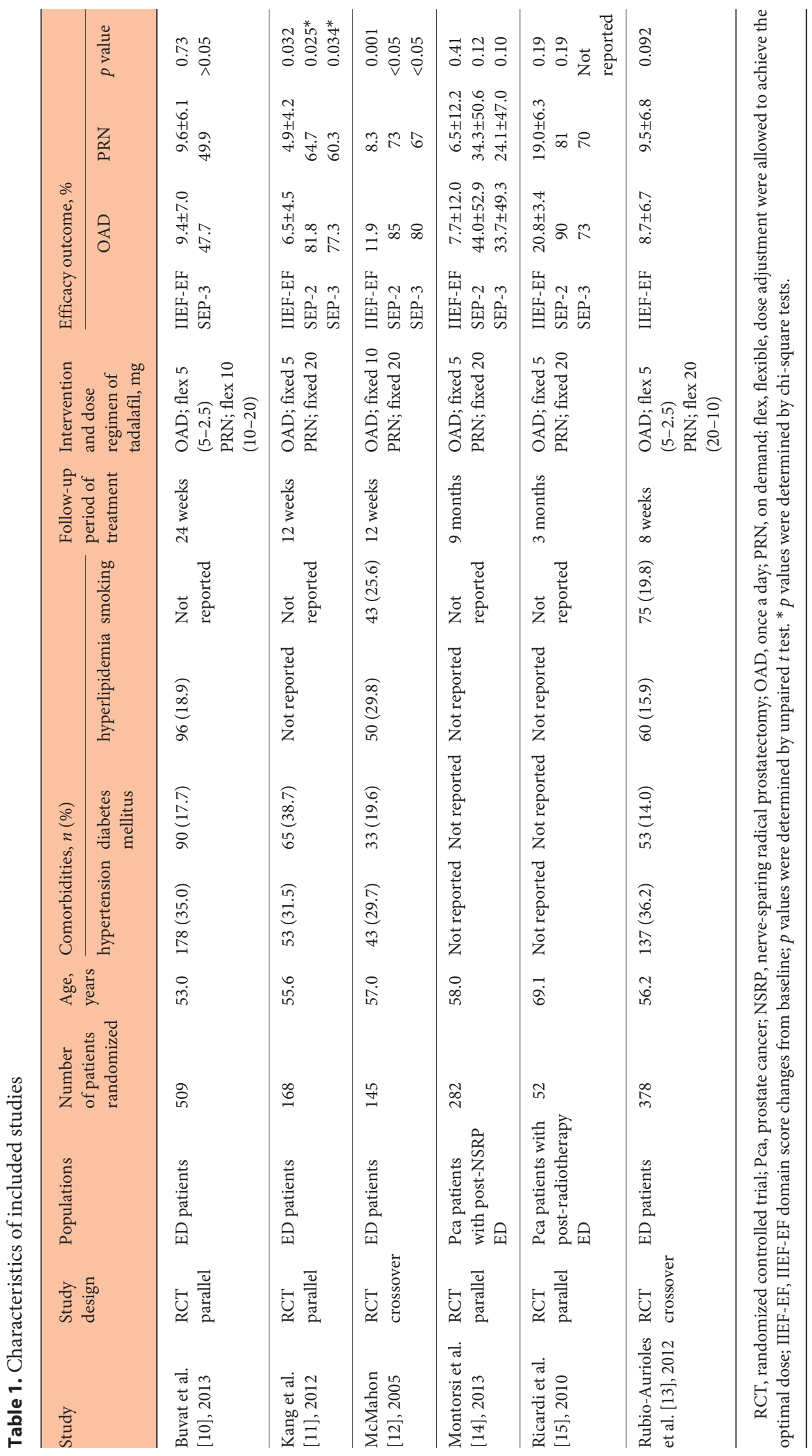


Fig. 2. a Risk of bias graph: review authors' judgements about each risk of bias item presented as percentages across all included studies. b Risk of bias summary: review authors' judgements about each risk of bias item for each included study.

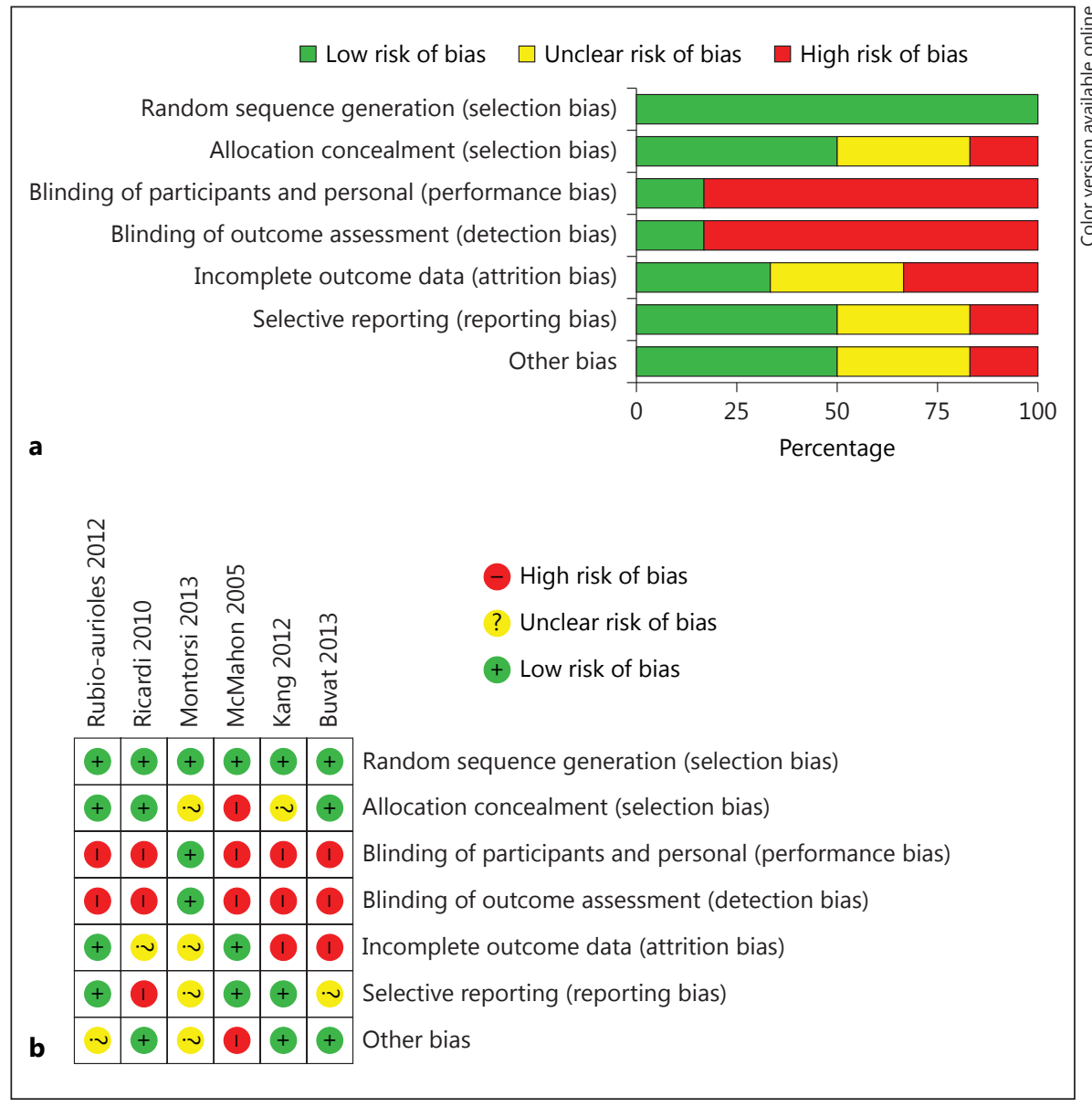

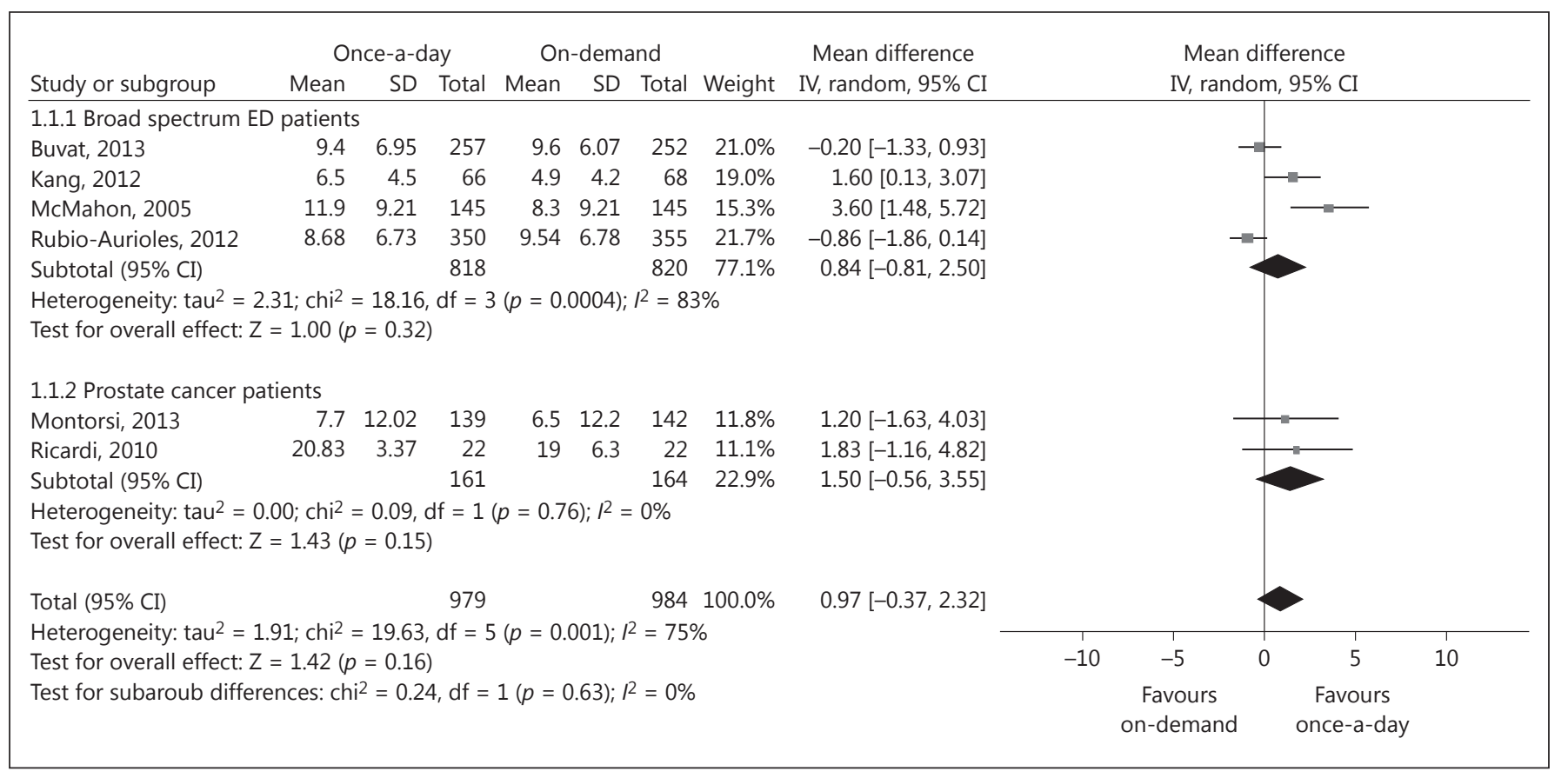

Fig. 3. Subgroup analysis of IIEF-EF domain score in ED patients using tadalafil once-a-day vs. on-demand.

Tadalafil Once-a-Day vs. Tadalafil On-Demand for ED
Urol Int 2017;99:343-352

DOI: $10.1159 / 000477496$ 


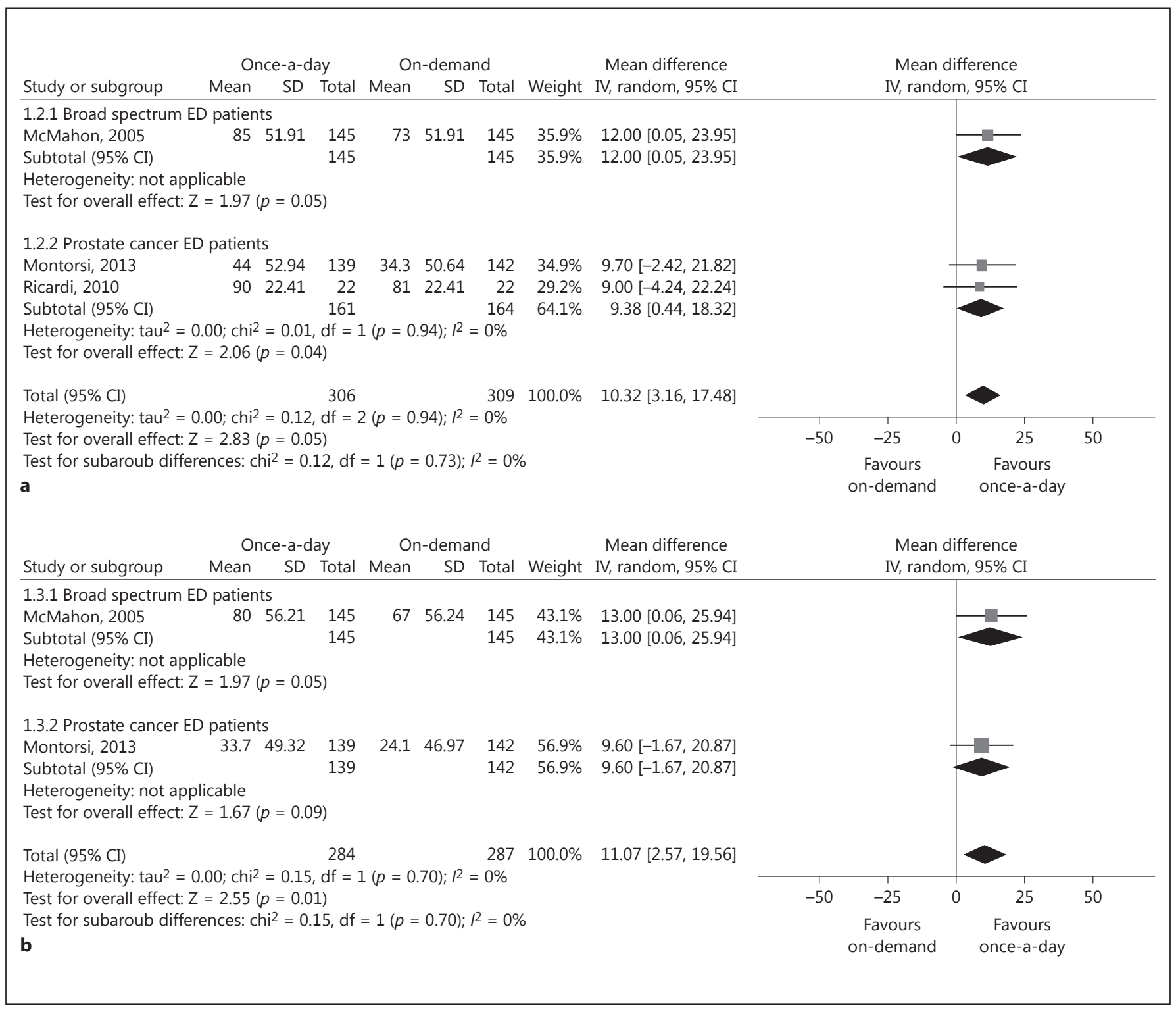

Fig. 4. a Subgroup analysis of SEP-2 in ED patients using tadalafil once-a-day versus on-demand. b Subgroup analysis of SEP-3 in ED patients using tadalafil once-a-day vs. on-demand.

ported that 1 patient in the once-a-day group had an ischemic stroke considered related to the study drug. One patient in the on-demand group died of suicide, which was considered unrelated to the study drug.

\section{Discussion}

Tadalafil is a selective PDE5 inhibitor with a half-life of $17.5 \mathrm{~h}$, which is longer than other available PDE5 inhibitors $[16,17]$. This property of tadalafil provides a wide window in which to engage in sexual intercourse. It also allows ED patients to choose once-a-day dosing regimen as an alternative to the conventional on-demand dosing regimen. This systematic review and quantitative meta-analysis integrated the data from RCTs that compared the efficacy and safety of different dosing regimen of tadalafil.

In the studies we reviewed, only one study had a blind design, which could decrease the accuracy of the outcomes. ED is a complex condition highly associated with psychological concerns. A blind study design would de- 


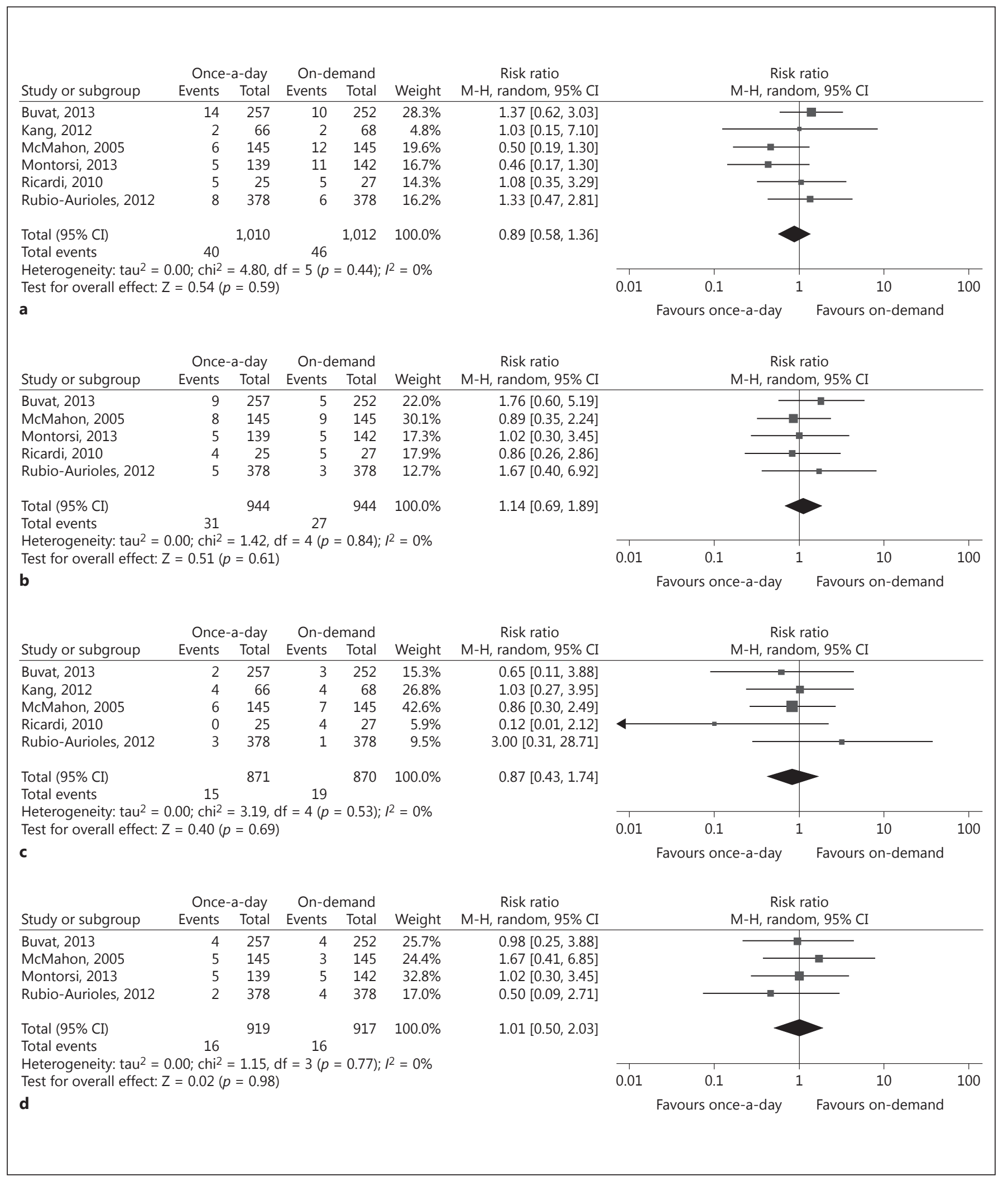

Fig. 5. Adverse events in ED patients using tadalafil once-a-day versus on-demand. a Headache; b dyspepsia; c flushing; d backache; e myalgia.

(For figure 5e see next page.)

Tadalafil Once-a-Day vs. Tadalafil On-Demand for ED
Urol Int 2017;99:343-352

DOI: $10.1159 / 000477496$ 


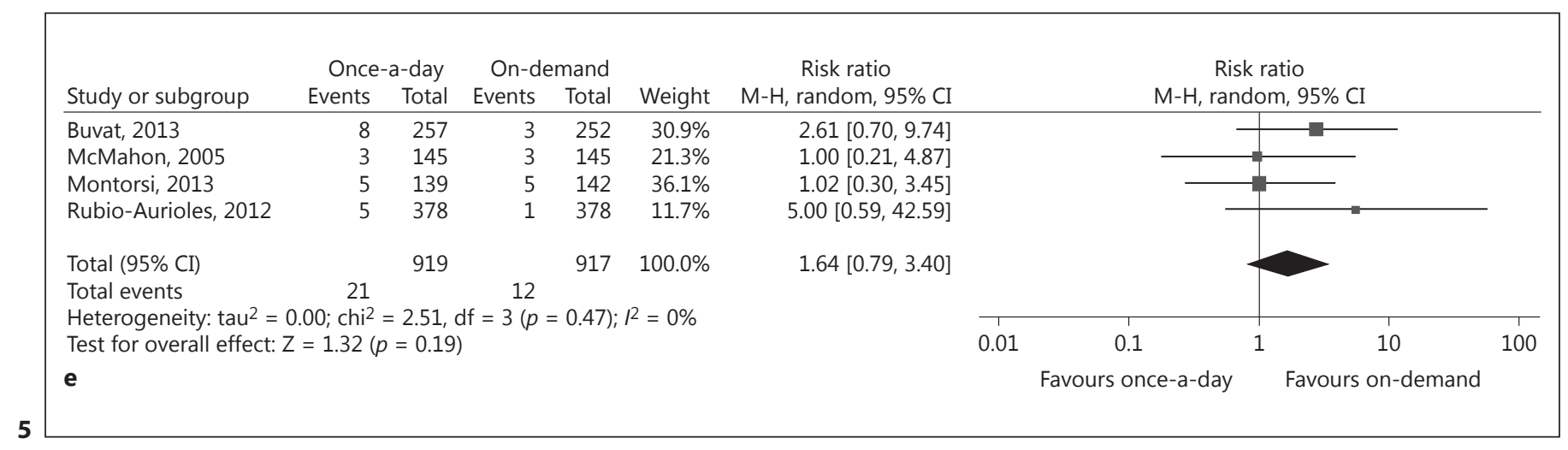

crease the accuracy of the real efficacy of intervention because it does not take psychosocial effects into consideration. An open-label design would decrease the internal validity, but it would be able to assess the psychosocial effects of the different dosing regimens. Blinding design would also ignore patients' experience of different dose regimens.

To assess efficacy, we evaluated the IIEF-EF domain score, SEP-2, and SEP-3. Improvement in the IIEF-EF domain score demonstrated that the efficacy of tadalafil once-a-day and on-demand were similar. SEP-2 and SEP-3 showed that once-a-day dosing regimen was superior to on-demand with statistical significance. We should note that only a few studies were included in the meta-analysis of SEP-2 and SEP-3. The result should be treated cautiously. In our integrated analysis of the efficacy outcome, tadalafil once-a-day might have the possibility to show a better outcome than on-demand. However, no conclusive evidence existed regarding the above matter.

For the on-demand dosing regimen, the association between drug intake and sexual intercourse could have psychological implications for patients. These psychological factors could affect patients' sexual intercourse performance, confidence, and quality of life. The length of the half-life of tadalafil could be an important factor affecting psychological concerns. In theory, a much longer effective plasma concentration could be maintained with a once-a-day dosing regimen than with an on-demand dosing regimen, allowing patients to feel ready for sexual intercourse at any time, and enabling them to separate drug intake from the act of sexual intercourse [17]. Based on these considerations, psychosocial outcomes appear to be associated with the dosing regimen of tadalafil. In studies conducted by Rubio-Aurioles et al. [13] and Hatzimouratidis et al. [18], Psychological and Interpersonal Relationship Scales were evaluated specifically, including sexual self-confidence, spontaneity, and time concerns. Both studies found that tadalafil once-a-day was superior to tadalafil on-demand with statistical significance in the time concerns domain. Improvements in sexual self-confidence and spontaneity were similar between once-a-day and on-demand dosing regimens.

Adverse events were relatively rare. In our review, headache and dyspepsia were the most common adverse events. The incidence of headache and dyspepsia was 4.3 and $2.9 \%$ respectively. The next common adverse events were flushing, backache, and myalgia, their incidence being $1.7,1.6$, and $1.6 \%$, respectively. In the integrated analysis of our study, once-a-day and on-demand dosing regimens showed similar complication rates. It is of interest to note that the once-a-day dosing regimen had a lower incidence of headache and flushing compared to the on-demand dosing regimen. One possible explanation was that headache and flushing were more likely to be associated with peak drug plasma concentration, which was much lower in the once-a-day dosing regimen than the on-demand regimen. In general, regardless of which dosing regimen of tadalafil was used, the adverse events were relatively rare and well tolerated.

Although there was no difference in efficacy and safety between tadalafil once-a-day and on-demand, patients had personal preferences for different dosing regimen for a variety of reasons. McMahon [12] reported that $72 \%$ of patients preferred the once-a-day dosing regimen and $28 \%$ patients preferred the on-demand dosing regimen. One possible explanation for the high percentage of patients who preferred the once-a-day dosing regimen is because free tadalafil was offered to $27.6 \%$ patients in this study, who were able to disregard the higher cost of taking 
tadalafil once a day. In a randomized study investigating treatment adherence to tadalafil once-a-day and on-demand in ED patients without previous exposure to PDE-5 inhibitors, Buvat et al. [10] found that the percentages of patients who switched treatments in the tadalafil once-aday group and the on-demand group were 43.2 and $31 \%$, respectively. The predominant reasons for preference or switching treatment included lack of efficacy, adverse events, sexual spontaneity, and time constraint, not wanting to take a pill regularly, concerned about the long-term risks of taking tadalafil once a day, and reporting a feeling that the drug controlled the patient's sexual life. In general, greater preference was shown for once-a-day dosing regimen than the on-demand regimen. However, both dosing regimens of tadalafil were chosen by a substantial number of patients.

Kim et al. [19] reported that tadalafil once-a-day significantly improved erectile function in $416 \mathrm{ED}$ patients who had mild to mild-moderate ED following maximum doses of PDE5 inhibitors on-demand. Forty percent of patients in the tadalafil 5-mg group returned to normal erectile function (IIEF-EF $\geq 26$ ) compared to $12 \%$ patients in the placebo group $(p<0.001)$. A study of Kim's demonstrated that once-a-day dosing regimen could be an alternative for patients who did not have success using an on-demand dosing regimen.

In summary, both tadalafil once-a-day and on-demand were effective and well tolerated. However, choosing the optimal dosing regimen for individual patients is difficult for a number of reasons, including drug efficacy, adverse events, ED etiology, previous ED medication, clinical features, sexual activity patterns, psychosocial factors, economic conditions, and personal preference. In clinical practice, we should choose a dosing regimen on the basis of individual characteristics and patient preference. Moreover, other dose regimens could be used as an alternative to previously tried regimens.

Our study had several limitations: (1) only one RCT used a blind study design; most of the RCTs included were open-label design, which could increase performance bias and detection bias. (2) We incorporated crossover design RCTs in meta-analysis by taking all outcomes from tadalafil once-a-day periods and those from on-demand periods as if the trial were parallel design trial of tadalafil once-a-day versus on-demand. This approach ignored the fact that the same patient appeared twice in the study, which gave rise to a unit-of-analysis error. (3) Two studies [10,13] did not report SEP-2. One study [13] did not report SEP-3. Standard error of SEP-2

Tadalafil Once-a-Day vs. Tadalafil On-Demand for ED of one study [11] could not be obtained. Standard error of SEP-3 of 3 studies $[10,11,15]$ could not be obtained. Therefore, only half of the included RCTs were usable for the meta-analyses of SEP-2 or SEP-3. (4) The follow-up time of RCTs varied, which may cause the heterogeneity. The lack of long-term follow-up RCTs was also an issue. The long-term efficacy and safety of different dosing regimens of tadalafil could not be evaluated. (5) Some studies included in this review were based on small sample sizes, which could cause the heterogeneity and selection bias.

\section{Conclusion}

Our study showed that tadalafil once-a-day and ondemand were effective and well-tolerated in both broadspectrum and prostate cancer ED patients. Once-a-day dose regimen might have the possibility to demonstrate a better efficacy outcome than on-demand regimen, and no statistically significant difference in safety was found between once-a-day and on-demand dose regimen. On the psychological scale of Psychological and Interpersonal Relationship Scales, the once-a-day dose regimen showed a superior time concerns outcome compared to the on-demand regimen. With respect to patient preference, the once-a-day dose regimen was considered better than the on-demand regimen. However, both dosing regimens were chosen by a substantial number of patients. The reasons for their preferences were complex, including drug efficacy, adverse events, clinical features, psychosocial factors, sexual activity patterns, and personal preference. Collectively, our study suggests that tadalafil once-a-day could be used as an alternative to tadalafil on-demand. However, we should offer the available dosing regimens to patients and help them choose according to these various preference factors on initial ED treatment.

\section{Acknowledgments}

This study was funded by the National Natural Science Foundation of China (grant No. 81370855). The funders had no role in study design, data collection, and analysis, decision to publish, or preparation of the manuscript.

\section{Disclosure Statement}

The authors have no conflicts of interest to declare. 


\section{Appendix 1. Search Strategy}

Medline (via Ovid)

1. randomized controlled trial.pt.

2. controlled clinical trial.pt.

3. randomized.ab.

4. placebo.ab.

5. randomly.ab.

6. trial.ab.

7. groups.ab.

8. or/1-7

9. exp animals/ not humans.sh.

10. 8 not 9

11. exp erectile dysfunction/

12. erectile dysfunction.tw.

13. or/11-12

14. exp tadalafil/

15. tadalafil.tw.

16. cialis.tw.

\section{References}

$1 \mathrm{NIH}$ Consensus Conference. Impotence. NIH Consensus Development Panel on Impotence. JAMA 1993;270:83-90.

2 Kubin M, Wagner G, Fugl-Meyer AR: Epidemiology of erectile dysfunction. Int J Impot Res 2003; 15:63-71.

3 Gratzke C, Angulo J, Chitaley K, Dai YT, Kim $\mathrm{NN}$, et al: Anatomy, physiology, and pathophysiology of erectile dysfunction. Curr Drug Targets 2015;16:411-419.

4 Saleh A, Abboudi H, Ghazal-Aswad M, Mayer EK, Vale JA: Management of erectile dysfunction post-radical prostatectomy. Res Rep Urol 2015;7:19-33.

5 Rosen RC, Kostis JB: Overview of phosphodiesterase 5 inhibition in erectile dysfunction. Am J Cardiol 2003;92:9-18.

6 Huang SA, Lie JD: Phosphodiesterase-5 (PDE5) inhibitors in the management of erectile dysfunction. P T 2013;38:407-419.

7 Choi H, Kim JH, Shim JS, Park JY, Kang SH, et al: Comparison of the efficacy and safety of 5-mg once-daily versus 5-mg alternate-day tadalafil in men with erectile dysfunction and lower urinary tract symptoms. Int $J$ Impot Res 2015;27:33-37.

8 Porst H, Gacci M, Büttner H, Henneges C, Boess F: Tadalafil once daily in men with erectile dysfunction: an integrated analysis of data obtained from 1913 patients from six randomized, double-blind, placebo-controlled, clinical studies. Eur Urol 2014;65:455-464.
17. or/14-16

18. once.tw.

19. per day.tw.

20. daily.tw.

21. oad.tw.

22. one time.tw

23. or/18-22

24. on demand\$.tw.

25. as need\$.tw.

26. prn.tw.

27. or/24-26

28. dose regimen $\$$.tw

29. dosing regimen $\$ . t w$

30. (drug adj4 administrat\$).tw

31. (tadalafil adj4 administrat\$).tw

32. (cialis adj4 administrat\$).tw

33. or $/ 28-32$

34.23 or 27 or 33

35.10 and 13 and 17 and 34
9 Higgins JP, Altman DG, Gøtzsche PC, Jüni P, Moher D, et al: The Cochrane Collaboration's tool for assessing risk of bias in randomised trials. BMJ 2011;343:d5928.

10 Buvat J, Büttner H, Hatzimouratidis K, Vendeira PA, Moncada I, et al: Adherence to initial PDE-5 inhibitor treatment: randomized open-label study comparing tadalafil once a day, tadalafil on demand, and sildenafil on demand in patients with erectile dysfunction. J Sex Med 2013;10:1592-1602.

11 Kang DH, Lee JY, Chung JH, Cho JM, Lee SH, et al: Comparison of efficacy for erectile function and lower urinary tract symptoms of tadalafil $20 \mathrm{mg}$ on-demand and $5 \mathrm{mg}$ once daily in patients with erectile dysfunction. Int J Clin Pract 2012;66:813-820.

12 McMahon C: Comparison of efficacy, safety, and tolerability of on-demand tadalafil and daily dosed tadalafil for the treatment of erectile dysfunction. J Sex Med 2005;2:415-425; discussion 425-427.

13 Rubio-Aurioles E, Porst H, Kim ED, Montorsi F, Hackett G, et al: A randomized open-label trial with a crossover comparison of sexual self-confidence and other treatment outcomes following tadalafil once a day vs. tadalafil or sildenafil on-demand in men with erectile dysfunction. J Sex Med 2012;9:14181429.

14 Montorsi F, Brock G, Stolzenburg JU, Mulhall J, Moncada I, et al: Effects of tadalafil treatment on erectile function recovery following bilateral nerve-sparing radical prostatectomy: a randomised placebo-controlled study (REACTT). Eur Urol 2014;65:587-596.
15 Ricardi U, Gontero P, Ciammella P, Badellino $S$, Valentino F, et al: Efficacy and safety of tadalafil $20 \mathrm{mg}$ on demand vs. tadalafil $5 \mathrm{mg}$ once-a-day in the treatment of post-radiotherapy erectile dysfunction in prostate cancer men: a randomized phase II trial. J Sex Med 2010;7:2851-2859.

16 Wang R, Burnett AL, Heller WH, Omori K, Kotera J, et al: Selectivity of avanafil, a PDE5 inhibitor for the treatment of erectile dysfunction: implications for clinical safety and improved tolerability. J Sex Med 2012;9: 2122-2129.

17 Wrishko R, Sorsaburu S, Wong D, Strawbridge A, McGill J: Safety, efficacy, and pharmacokinetic overview of low-dose daily administration of tadalafil. J Sex Med 2009;6: 2039-2048.

18 Hatzimouratidis K, Buvat J, Büttner H, Vendeira PA, Moncada I, et al: Psychosocial outcomes after initial treatment of erectile dysfunction with tadalafil once daily, tadalafil on demand or sildenafil citrate on demand: results from a randomized, open-label study. Int J Impot Res 2014;26:223-229.

19 Kim ED, Seftel AD, Goldfischer ER, Ni X, Burns PR: A return to normal erectile function with tadalafil once daily after an incomplete response to as-needed PDE5 inhibitor therapy. J Sex Med 2014;11:820-830. 